\title{
Design of the Sine Wave Generating Circuit Based on the ATmega128A
}

\section{Microcontroller}

\author{
JinYang $\mathrm{Xia}^{1, \mathrm{a}} \quad$ YanPing Wang*2 \\ ${ }^{1.2}$ Beijing institute of fashion technology, China \\ a Email: 1171819892@qq.com
}

Key words: ATmega128A; Sinusoidal Pulse Width Modulation; three -phase sine wave; zero point matching; phase control

Abstract. This paper mainly discussed how to generate three-phase sine wave by ATmega128A single chip microcomputer. The paper describes the principle of sine pulse width modulation and pulse width algorithm of SPWM wave in detail, briefly introduces the structure and the function of ATmega128A microcontroller, and describes the functional modules used in the lab in detail. The hardware part analyses several modules of the principle diagram of the circuit boards, describes the process of drawing PCB diagram and the design of the second order filter circuit. The software part is designed in detail based on the previous theory.

\section{Introduction}

With the development of inverter technology in various fields, researches on inverter control has become an important research topic. At present, it is widely used in the inverter power supply with sinusoidal pulse width modulation. For example, using the integrated circuit, is one of way to get the signal. SPWM control circuit is simple and easy control, but the feedback control of system can not be ignored.

\section{Sine Pulse Width Modulation Principle}

The narrow pulse with the same impulse, but in different shapes, is basically the same as that in the process of inertia. According to the principle of equivalent area $\mathrm{N}$, the amplitude of the rectangular pulse width waveform is consisting with equivalent sine wave. The frequency of PWM wave can be obtained by setting the frequency of sine wave:

$$
f_{p w m}=N \times f_{c}
$$

The method of determining the pulse width, with the intersection of a sine wave and a triangular wave, is called the natural sampling method. Figure1 is the intersection of sine wave and triangle wave, for the duration of the pulse width. $\triangle D E C \sim \triangle A F C$ can be used to figure out the relation between triangle wave crest value and sine wave amplitude, by the: 


$$
\frac{2 U_{C M}}{T_{C} / 2}=\frac{U_{C M}+U_{R M} \sin \left(\mathrm{wt}_{\mathrm{A}}\right)}{t_{2}^{\prime}}
$$

In the same way:

$$
\frac{2 U_{C M}}{T_{C} / 2}=\frac{U_{C M}+U_{R M} \sin \left(\mathrm{wt}_{\mathrm{B}}\right)}{t_{2}^{\prime}}
$$

The finishing:

$$
t_{2}=t_{2}^{\prime}+t_{2}^{\prime \prime}=\frac{T_{C}}{2}\left[1+\frac{M}{2}\left(\sin w t_{A}+\sin w t_{B}\right)\right] \quad\left(M=\frac{U_{R M}}{U_{C M}}\right)
$$

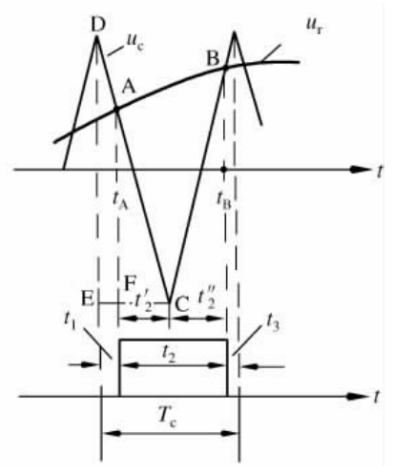

Figure 1. Natural sampling method for generating SPWM wave

\section{Hardware Circuit PCB Board Design}

The whole process is: the new PCB file, into the PCB board editing interface, setting the PCB board for the four laminates, power layer segmentation, respectively, VCC and VDD. The PCB plate of the rectangular border will be drown and joined into the components and the network table, which includes component layout and circuit board wiring.

After importing components and network table, all the components will be stacked together, and staying outside the PCB board. The layout of the components will not only affect the difficulty of the work of the following wiring, but also the actual situation of the circuit board. The layout of components is divided into two kinds of layout including automatic layout and manual layout. According to the actual situation, we need to carry out automatic layout, so the components are automatically distributed to the PCB board, which can speed up the layout of components. In order to save space, the patch capacitance and resistance are put on the bottom. And special attention should be paid to the capacitor line which can not be too long.

Circuit board wiring is divided into automatic and manual routing. But after the automatic routing, circuit surface is always in disorder, not clean, and has mangy line bypass. So manual routing becomes the final choice. In the late period of circuit board wiring, the distribution of components is not reasonable, the way to solve this problem is to readjust the distribution of components, and increase the workload.Thus it can be seen that the distribution of components is very important to the premise. The final PCB design is shown in figure 2. 


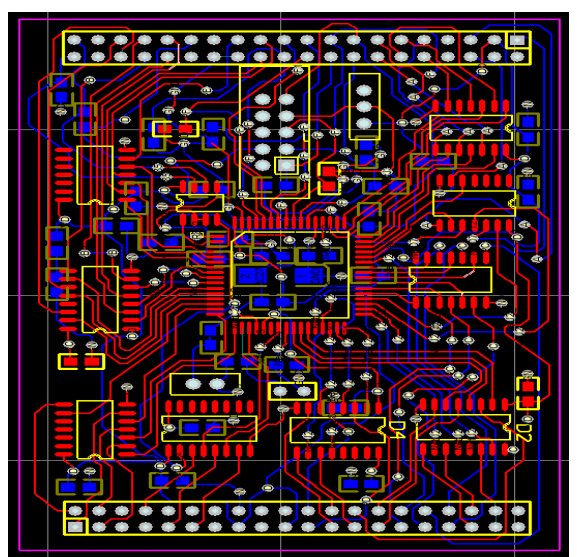

Figure 2. PCB design

\section{Software Design}

Main program design. The software programming part mainly includes: the main function, the port initialization function, the timer initialization function, the overflow interrupt function and the capture interrupt function.

Port initialization: according to ATmega128A microcontroller I/O port description, the pin I/O port register need to be set up, ensuring they can work normally. Three output pins are used as the output pin of the three SPWM wave, and one input pin is used as the $50 \mathrm{HZ}$ square wave input pin.

Timer initialization: according to the results of the previous derivation, two 16-bit timer would be used in this experiment. The TOP value is 1240, and the ICR1 register can be selected to define the TOP value. At the same time, the OCR1A/B/C is used to output the PWM wave, and the ICR3 is used to capture interrupt. The registers, are required to configuration.

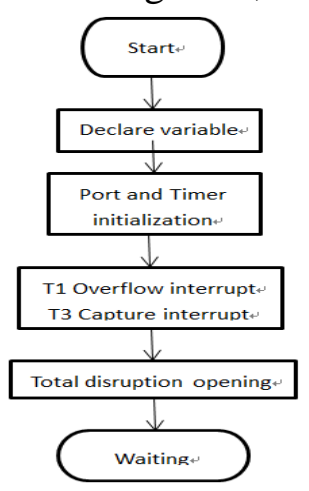

Figure 3.Main program flow chart

Overflow interrupt program design. The function of the overflow interrupt is to change the value of the output compare register. In the main function, the T1 overflow interrupt is accessible, and the total interrupt is accessible. When the counter value rises to the TOP value, T1 interrupt flag would be set to 1 , then entering the interrupt function, meanwhile $\mathrm{T} 1$ interrupt flag automatically would be cleared to 0 .

Capture interrupt program design. The function of the capture interrupt is to achieve zero matching, to realize phase shift detection, and to make the mark. In the timer initialization, T3 is set up as rising edge. Between the main function, the capture interrupt and the total interrupt are accessible. Through pin ICP3, 50HZ square wave can be input, when a rising edge is captured, 
the input capture flag bits would be set as high level, the input capture can be carried out, and the interrupt flag is cleared automatically. In the interrupt function, the table pointer is forced to point at the first number, so that the first pulse width of the PWM wave in each cycle can correspond to the sine wave to achieve zero matching.

The other two square wave are received by A1:0 pin, when entering the capture interrupt, the level of A pin can be read to estimate whether there is a phase change or not. If the value of A pin is $0 \times 01$, then the phase is not changed; if the value of A pin is $0 \times 10$, it indicats that the two square wave phase swaps. In the overflow interrupt, the level of A pin decides whether to exchange OCR1B and OCR1C's checking table pointer.

\section{Sine Wave Generation}

After two - step filter, the SPWM wave is obviously asymmetric. There may be two reasons for the analysis: 1 . The SPWM pulse width calculation has error; 2 , the filtering parameter RC is not appropriate. Solution is to derivate the principle to the formula to confirm that the formula is not wrong. So the calculation must exist problems, and the problem should be the filter parameter design which is not appropriate. The second step ensures that the cut-off frequency is not modified, except the RC value and the capacity value. Thus, the sine wave would be shown on output screen which is same to Figure 4.

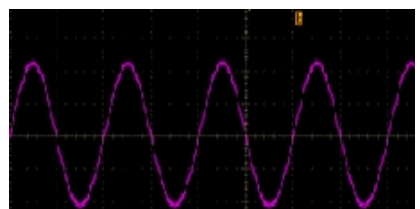

Figure 4. Output sine wave

\section{Conclusion}

With a whole system design, I am familiar with the integrated development environment and the combined use of development environment, including programming, debugging and downloading. The system study covers the the process of the PWM wave of the timer / counter used in Atmega128A microcontroller, the process of the interrupt processing, and the understanding of the principle of the pulse width calculation formula. Through this experiment, I clearly recognize that my own shortcomings, in future works, and I will continue to work hard.

\section{Acknowledgment}

This work is supported by Beijing Institute of Fashion Technology Project support (NO.ZD-1407), all support is gratefully acknowledged.

\section{Reference}

[1] JianLiang Shen, WenHong Zhao, YuKun Jia, HuaDong Wang, KeJia Hu.ATmega128 MCU entry and improve. 2009

[2] Zeng Yang Leng, YiRong Xu. Power Electronics Technology (Third Edition). 2012

[3] Mei Li, XiaoDong Han, Jie Zhang. Power electronics technology. 2012 
[4] Li Zhang, JingBo Zhao, Jiang Chang. Circuit design and plate making 99SE PROTEL from entry to the master. 2012

[5] Lucien Ngalamou, Leary Myers. Digital SPWM synthesis for the design of single phase inverters. International Journal of Electronics.2008 